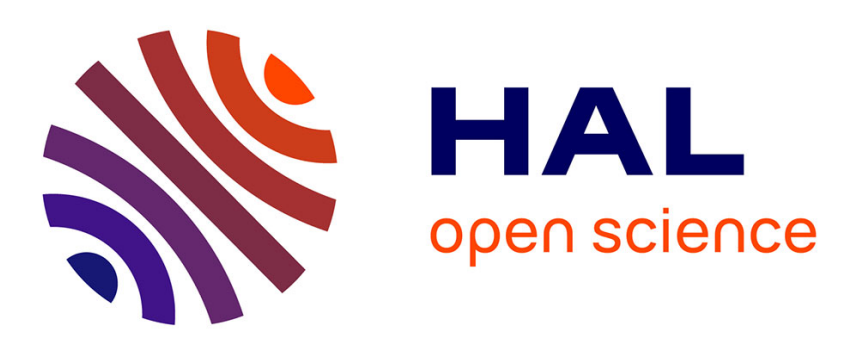

\title{
Implementation of high resolution diffractive optical elements on coupled phase and amplitude spatial light modulators
}

Christophe Stolz, Laurent Bigue, Pierre Ambs

\section{- To cite this version:}

Christophe Stolz, Laurent Bigue, Pierre Ambs. Implementation of high resolution diffractive optical elements on coupled phase and amplitude spatial light modulators. Applied optics, 2001, 40 (35), pp.6415-6424. 10.1117/12.504948 . hal-00955421

\section{HAL Id: hal-00955421 https://hal.science/hal-00955421}

Submitted on 4 Mar 2014

HAL is a multi-disciplinary open access archive for the deposit and dissemination of scientific research documents, whether they are published or not. The documents may come from teaching and research institutions in France or abroad, or from public or private research centers.
L'archive ouverte pluridisciplinaire HAL, est destinée au dépôt et à la diffusion de documents scientifiques de niveau recherche, publiés ou non, émanant des établissements d'enseignement et de recherche français ou étrangers, des laboratoires publics ou privés. 


\title{
Implementation of high-resolution diffractive optical elements on coupled phase and amplitude spatial light modulators
}

\author{
Christophe Stolz, Laurent Bigué, and Pierre Ambs
}

\begin{abstract}
We propose the optical implementation of diffractive optical elements onto electrically addressed liquidcrystal spatial light modulators. We compare the classic implementations onto amplitude-only or phaseonly domains with the implementations onto coupled phase and amplitude (spiral) domains. We demonstrate that the coupling between amplitude and phase provides a trade-off between diffraction efficiency and the signal-to-noise ratio in the reconstruction. Furthermore, when investigating the influence of the maximum dephasing on phase domains and spiral domains through the use of optimal trade-off design, we show that phase-only domains with limited maximum dephasing can provide satisfactory performance. Finally, optical implementations are provided. (c) 2001 Optical Society of America

OCIS codes: $\quad 090.1760,090.1970,230.6120,230.3720$.
\end{abstract}

\section{Introduction}

Nowadays, the use of diffractive optical elements (DOEs) has become more and more important in many applications because of their smaller size compared with classic refractive elements and their low cost and high quality owing to their innovative computing and fabrication methods. However, some applications such as correlation filters and adaptive optics ${ }^{1}$ require a real-time adaptation of the DOE. To implement such optical functions, we must use appropriate electro-optical devices. This is why here we try to use commercially available twisted nematic (TN) liquid-crystal (LC) spatial light modulators (SLMs), which have been commonly available since the mid-1980's. These devices have proved particularly interesting because of their reduced cost

When this research was performed, the authors were with Ecole Supérieure des Sciences Appliquées pour l'Ingénieur-Mulhouse, Université de Haute Alsace, Laboratory Modeling Intelligence Process Systems, 12 rue des Frères Lumière, 68093 Mulhouse cedex France. C. Stolz is now with the Institut Universitaire de Technologie Le Creusot, Université de Bourgogne, 12, rue de la Fonderie, 71200 Le Creusot, France. The e-mail addresses of the authors are stolz@iutlecreusot.u-bourgogne.fr, L.Bigue@uha.fr, and P.Ambs@uha.fr. L. Bigué is the corresponding author.

Received 18 January 2001; revised manuscript received 29 June 2001.

0003-6935/01/356415-10\$15.00/0

(C) 2001 Optical Society of America and their high resolution (XGA devices, i.e., with $1024 \times 768$ pixels, are now available ${ }^{2}$ ) and have been used for the implementation of pattern-recognition filters ${ }^{3,4}$ and DOEs. ${ }^{5}$ They inherently present a coupled modulation between amplitude and phase, but a binary-amplitude mode or a phase-mostly mode can be reached. Amplitude-mostly modes have seldom been reported in the literature dedicated to these devices. In contrast, phase-mostly modes are commonly used ${ }^{6}$ because they are known to provide reconstructions as accurate as their amplitude-only (hardly reachable) counterparts. Coupled phase and amplitude domains have never been deeply investigated. In this paper we propose an objective comparison of all these domains through the use of the optimal trade-offs (OT) framework while exploring the influence of maximum dephasing (i.e., the maximum phase modulation that can be achieved) provided by each domain. In Section 2 we recall the optimal trade-off design principle. In Section 3 we present the optical characterization of a TN-LC device with the determination of its reachable coding domains. In Section 4 we present simulations of test DOEs onto various coding domains, either theoretical domains or experimental ones, and we provide the corresponding optical reconstructions.

\section{Review of Optimal Trade-Offs Framework}

The concept of OTs was presented to the optical community by Réfrégier. ${ }^{7}$ It was originally applied to 


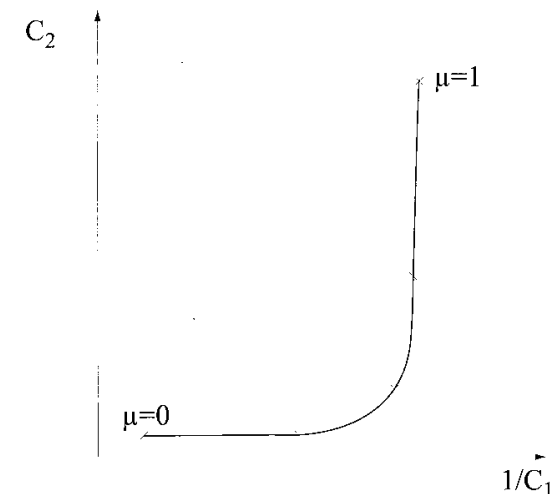

Fig. 1. Typical OCC [i.e., locus of points $\left(C_{1}(\mu), C_{2}(\mu)\right)$ ] when $C_{1}$ and $C_{2}$ are to be minimized. $\mu=0$ corresponds to optimizing only $C_{2}$ and $\mu=1$ corresponds to optimizing only $C_{1}$.

correlation filters and was then extended by Legeard et al. ${ }^{8}$ to diffractive optical elements. OT DOEs ${ }^{8}$ are DOEs that, given two antagonistic criteria $C_{1}$ and $C_{2}$, optimize criterion $C_{1}$ by taking account of a weighting by $C_{2}$. To obtain such trade-offs, we work with the criterion formed with the linear combination of the two criteria when $0 \leq \mu \leq 1$ :

$$
C=\mu C_{1}+(1-\mu) C_{2}
$$

We then obtain a point $\left(C_{1}(\mu), C_{2}(\mu)\right)$ in the space of all possible trade-offs associated with our criteria. The locus of all these points is the optimal characteristics curve (OCC). Its typical shape is shown in Fig. 1 in the case when we minimize $C_{1}$ and $C_{2}$ or equivalently maximize $1 / C_{1}$ and $1 / C_{2}$ and shows the relation between $1 / C_{1}$ versus $C_{2}$ (useful for our future DOE design).

In the design of DOEs, the classic criteria that are considered are the amplitude signal-to-noise ratio $\left(\mathrm{SNR}_{\mathrm{a}}\right)$ and the diffraction efficiency $\eta$. Equations (2)-(4), from Ref. 8, express $\operatorname{SNR}_{\mathrm{a}}$ and $\eta$ when $f$ is the desired reconstruction, $g$ is the obtained reconstruction, $\mathfrak{I}$ is the working window (region of interest) of the reconstruction plane, and $\mathrm{ERR}_{\mathrm{a}}$ is the normalized amplitude error,

$$
\mathrm{SNR}_{\mathrm{a}}=\frac{1}{\mathrm{ERR}_{\mathrm{a}}}=\frac{\sum_{(q, r) \in \Im}|f(q, r)|^{2}}{\sum_{(q, r) \in \Im}\left[|f(q, r)|-c_{a}|g(q, r)|\right]^{2}},
$$

where

$$
c_{a}=\frac{\sum_{(q, r) \in \Im}|f(q, r)||g(q, r)|}{\sum_{(q, r) \in \mathfrak{I}}|g(q, r)|^{2}}
$$

is the scale factor minimizing $\mathrm{ERR}_{\mathrm{a}}$ for a given $f$ and g.

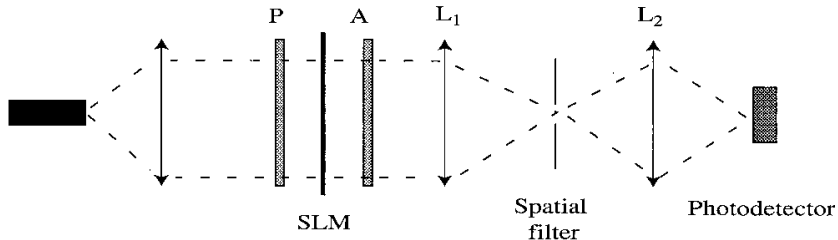

Fig. 2. Optical system used for the characterization of the SLM. $\mathrm{P}$ and $\mathrm{A}$, the polarizer and the analyzer, respectively.

$\eta$ is expressed as

$\eta=\frac{\text { desired reconstruction energy }}{\text { incident light energy }}=\frac{\sum_{(q, r) \in \Im}|g(q, r)|^{2}}{M N}$,

where $M N$ is the number of pixels in the DOE.

In this paper the direct binary search ${ }^{9}$ (DBS) algorithm is used. This iterative technique is based on a Monte Carlo search and minimizes a criterion. The criterion for DOE computation is usually $\mathrm{SNR}_{\mathrm{a}}$, but a criterion consisting of a linear combination of $\mathrm{SNR}_{\mathrm{a}}$ and optical efficiency is also possible, ${ }^{8}$ opening a multicriteria perspective. Although DBS gives excellent results, it requires high computation times; therefore another multicriteria technique, the multicriteria iterative Fourier transform algorithm (MIFTA), has been proposed. ${ }^{10}$ Nevertheless, in that the comparison of both techniques is not within the scope of this paper, only multicriteria DBS will be used because it provides slightly better results than the multicriteria iterative Fourier transform algorithm.

$\mathrm{SNR}_{\mathrm{a}}$ and optical efficiency are antagonistic. That is why the concept of OTs provides a convenient framework for studying DOEs. We used the following criterion, to be minimized8:

$$
C=\mu \frac{1}{\eta}+(1-\mu) \mathrm{ERR}_{\mathrm{a}} .
$$

To draw OCCs, we used a set of ten images with various characteristics (interconnection patterns, random distributions, classic images, etc.) with a size of $32 \times 32$ pixels to compute DOEs of $128 \times 128$ pixels and to draw OCCs. These images are used for all the OCCs shown in the next sections. The OT framework can be used for the comparison of coding domains (this is the purpose of this paper) or the comparison of coding techniques as suggested in Ref. 8.

Before exploring the different coding domains used in this study, Section 3 describes the characterization of our TN-LC SLM.

\section{Optical Characterization of a Twisted Nematic Light-Crystal Spatial Light Modulator}

The SVGA1 $(800 \times 600)$ device from CRL Opto, ${ }^{2}$ which must be placed between a polarizer-analyzer pair (Fig. 2), was optically characterized. This device is of $24-\mu \mathrm{m} \times 26-\mu \mathrm{m}$ pixel size with a $33-\mu \mathrm{m} \times$ 
Table 1. Equation System Used for Amplitude Measurements ${ }^{a}$ When a Gray-Level Pattern Is Displayed on the SLM

\begin{tabular}{cccc}
\hline Configuration & $\psi_{P}$ & $\psi_{A}$ & Intensity \\
\hline 1 & 0 & 0 & $c^{2}\left(f^{2}+g^{2}\right)$ \\
2 & 0 & $\pi / 4$ & $c^{2}(0.5+f h+g j)$ \\
3 & 0 & $\pi / 2$ & $c^{2}\left(h^{2}+j^{2}\right)$ \\
4 & $\pi / 4$ & $-\pi / 4$ & $c^{2}\left(h^{2}+g^{2}\right)$ \\
5 & $\pi / 4$ & 0 & $c^{2}(0.5-f h+g j)$ \\
6 & $\pi / 4$ & $\pi / 4$ & $c^{2}\left(f^{2}+j^{2}\right)$ \\
\hline
\end{tabular}

${ }^{a}$ From Ref. 13.

$33-\mu \mathrm{m}$ pitch, and its electronic board is designed to be directly driven by a super video graphics array (SVGA) video signal delivered by a computer. Such devices cannot operate at frame rates higher than a few tens of hertz because of the low commutation speed of TN LCs. The device characterization was performed through the determination of Jones matrices ${ }^{11-14}$ : This technique provides a model for the LC matrix and then allows the user to predict the device behavior for given configurations of the polarizer and analyzer. Historically, this characterization was performed with a Mach-Zehnder interferometer, ${ }^{12,15}$ but the data obtained for the phase measurements were often noisy and hard to exploit, ${ }^{16}$ as was pointed out in Ref. 11 .

Thus for a given TN-LC cell (i.e., a pixel) sandwiched between an analyzer and a polarizer with respective azimuth angles $\psi_{a}$ and $\psi_{b}$, the output Jones vector is calculated to be $^{12}$

$$
\left(\begin{array}{c}
x_{\text {out }} \\
y_{\text {out }}
\end{array}\right)=P\left(\psi_{A}\right) J P\left(\psi_{P}\right)\left(\begin{array}{l}
x_{\text {in }} \\
y_{\text {in }}
\end{array}\right)
$$

where

$$
\left(\begin{array}{l}
x_{\text {in }} \\
y_{\text {in }}
\end{array}\right)
$$

denotes the incident Jones vector, $J$ is the Jones matrix of the LC cell, and $P(\psi)$ is the Jones matrix of a polarizer azimuth angle $\psi . \quad J$ is described by

$$
J=c \exp (-\mathrm{i} \beta)\left(\begin{array}{cc}
f-\mathrm{i} g & -h-\mathrm{i} j \\
h-\mathrm{ij} & f+\mathrm{i} g
\end{array}\right),
$$

where $c$ represents intensity loss by surface reflections, etc., $\beta$ represents the birefringence, i represents the imaginary unit, and $P(\psi)$ is expressed as

$$
P(\psi)=\left(\begin{array}{cc}
\cos ^{2} \psi & \sin \psi \cos \psi \\
\sin \psi \cos \psi & \sin ^{2} \psi
\end{array}\right)
$$

Fernández-Pousa et al. ${ }^{17}$ showed that ideal LC cells have simpler expressions of their Jones matrix, but because our LC modulator is not supposed to be perfect, we prefer to consider the general form of Eq. (7).

To fully characterize the modulator, we have to determine its Jones matrix $J$. To achieve this, the technique used in this paper, as suggested by Colin, ${ }^{13}$ first consists in measuring, for various gray-level values, the amplitude transmittance of the SLM using
Table 2. Expression of the Phase Retardation for the Six Considered Configurations $^{\alpha}$ When a Ronchi Ruling Is Displayed on the SLM

\begin{tabular}{cccc}
\hline Configuration & $\psi_{P}$ & $\psi_{A}$ & Dephasing $\phi_{2}-\phi_{1}$ \\
\hline 1 & 0 & 0 & $\beta+\arctan (g / f)$ \\
2 & 0 & $\pi / 4$ & $\beta+\arctan [(g+j) / f+h)]$ \\
3 & 0 & $\pi / 2$ & $\beta+\arctan (j / h)$ \\
4 & $\pi / 4$ & $-\pi / 4$ & $\beta+\arctan (g /-h)$ \\
5 & $\pi / 4$ & 0 & $\beta+\arctan [(g+j) /(f-h)]$ \\
6 & $\pi / 4$ & $\pi / 4$ & $\beta+\arctan (j / f)$ \\
\hline
\end{tabular}

${ }^{a}$ From Ref. 13.

the six different polarizer-analyzer configurations listed in Table 1, each configuration giving a particular equation of the transmittance.

The first series of intensity measurements concerns the central order of the diffraction pattern that is due to the SLM electrode grid when we display a uniform gray-level image.

For each gray level, the solution of the six-equation system of Table 1 corresponding to these amplitude measurements gives the absolute value of $f, g, h$, and $j$ and the products $f h$ and $g j$. To determine the entire Jones matrix, i.e., the signs of $f, g, h$, and $j$, we have to perform additional experiments for each gray level. To do so, we display a Ronchi ruling ${ }^{11}$ (a binary grating with a 50\% duty cycle) on the SLM and measure the intensity of the central order and of the $1 / 2$ order (supplementary diffraction order due to the Ronchi grating only and not to the SLM electrode grid) of its diffraction pattern, for the six configurations listed in Table 2. From these measured intensities, we can compute the dephasing between the two gray levels of the grating. Equation (9) shows the expression of the intensity of the $(p / 2)$ th diffraction order, where $m_{1}$ and $m_{2}$ are the intensities of the corresponding zero order when the two gray levels are displayed independently, as in the previous series of measurements. $\quad \phi_{2}-\phi_{1}$ is the dephasing between the two gray levels, $N$ is the number of columns of the grating, and $a$ is the width of each column (i.e., the modulator pitch), ${ }^{11}$

$$
\begin{aligned}
\left|A\left(\frac{p}{2}, 0\right)\right|^{2}= & \frac{N^{2}}{4} a^{2} \operatorname{sinc}^{2}\left(\frac{a p}{2 b}\right)\left[m_{1}{ }^{2}+m_{2}{ }^{2}\right. \\
& \left.+2 m_{1} m_{2} \cos \left(\phi_{1}-\phi_{2}\right)\right],
\end{aligned}
$$

where $b$ is the grating total width. For a better accuracy, redundant measures are performed for $p=0$ and $p=1$. This technique is supposed to be valid only if the phase is constant all over the pixel. This has not been verified, because such an investigation is not within the scope of this paper, ${ }^{18}$ but experimental results in good agreement with results obtained with a Mach-Zehnder interferometer for a similar device have been obtained.15

The dephasing values $\phi_{2}-\phi_{1}$ that are obtained from Eq. (9) can then be input in the six-equation system described in Table 2, and, finally, the signs of $f, g, h$, and $j$ can be determined.

Performing measurements for both intensity and 


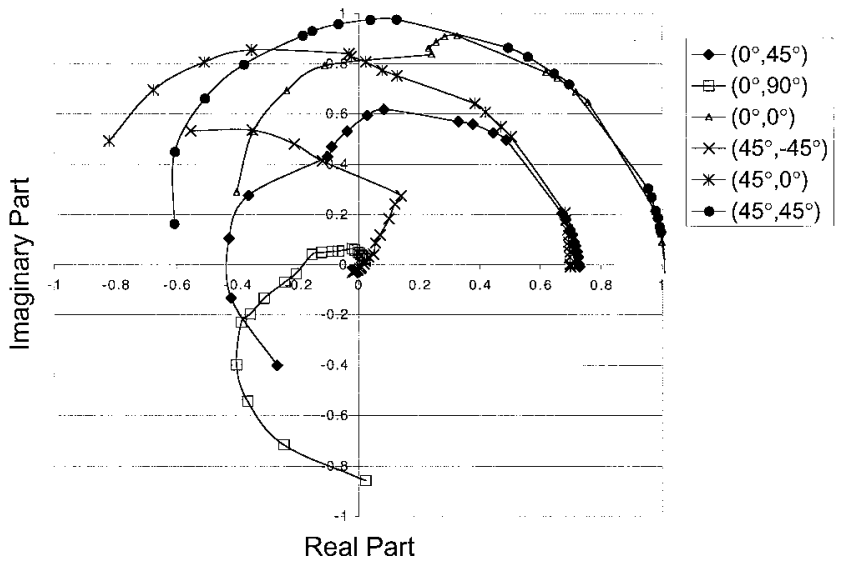

Fig. 3. Six coding domains resulting from the characterization at $633 \mathrm{~nm}$ of the SLM, corresponding to six polarizer-analyser configurations.

phase figures for six configurations provides redundant information for the only five parameters that we have to determine, which allows us to remove much noise. The measurements were performed at a wavelength of $633 \mathrm{~nm}$, for the arbitrary number of 27 gray values, ranging from 0 to 255 , with an increment of ten gray levels between them.

The six polarizer-analyzer configurations used in the experiments give quite different behaviors of the device. Figure 3 shows the curves obtained for the six configurations used during the characterization. The same experiment was performed either at 532 $\mathrm{nm}$ with this SLM or at $532 \mathrm{~nm}$ with a second SVGA1 SLM from CRL Opto, and the results obtained are close to those presented in Fig. 3. This suggests the possibility of use of the characteristic curves obtained here for a SLM of the same type with a reduced estimation error.

We noticed that at $633 \mathrm{~nm}$ the maximum dephasing was $3 \pi / 2$, which is lower than the ones obtained with the previous generation of devices that were thicker. Labastida et al. ${ }^{15}$ reported such a behavior with a similar device.

After this characterization, we will be able to use the model of the SLM for the choice of coding domains suitable for implementing DOEs. The LC optical response does not vary linearly with the applied voltage; therefore the coding domains will not be chosen with equal voltage increments but rather with driving voltages that allow a good exploration of the coding curve.

\section{Comparison of Coding Domains for Implementation of Diffractive Optical Elements}

In this section we will compare coding domains with various maximum dephasing. We will first consider theoretical coding domains and then coding domains achievable with our SVGA1 SLM, as described in Section 3. For all the domains used, we choose eight quantization levels to get good quality. All the OCCs are computed with DBS: The diffractive elements are directly computed onto the eight-level cod- ing domains, and no extra quantization operation is to be performed. For each complex value to be encoded, the search over the eight points of the coding domains is performed to determine which point will minimize the criterion expressed in Eq. (5).

\section{A. Theoretical Domains}

\section{Phase Domains}

We computed the OCCs of phase domains with respective maximum modulation $\pi / 2, \pi, 3 \pi / 2$, and $2 \pi$ (Fig. 4). As we expected intuitively, we immediately notice the higher values of the diffraction efficiency when the phase modulation increases. This fact can be explained by the progressive decrease in the central order when the phase modulation increases toward $2 \pi$.

Each point of the curve corresponds to a unique $\mu$ factor. At the $\mu=0$ point, only the $\mathrm{SNR}_{\mathrm{a}}$ is optimized, and lower diffraction efficiency is obtained.

We note that the differences in diffraction efficiency between the $3 \pi / 2$ and the $2 \pi$ domains are not very important and do not exceed $10 \%$ for equivalent values of the signal-to-noise ratio.

From these curves we can get an interval (dependent on $\mu$ ) for which the trade-off between the criteria can be considered as relevant. Within this range, efficiency greatly increases, whereas error does not significantly increase. Beyond this range, efficiency remains quasi uniform.

\section{About the Use of Spiral Domain}

Considering the good results provided by phase domains, we may wonder if it is necessary to investigate the performance of spiral domains, i.e. of domains for which amplitude and phase modulations are highly coupled (actually, if the phase is a linear function of the amplitude, we obtain a spiral in the complex plane). The first reason for doing so is that it is not yet possible to produce multilevel pure-phase elements, except with laboratory devices: Broomfield et al. ${ }^{19}$ proposed a cascade of binary ferroelectric LC SLMs, and Davis et al. ${ }^{20}$ described a VGA parallelaligned nematic LC SLM, but the only dynamic multilevel pure-phase device commercially available is the Hamamatsu optically addressed parallel-aligned SLM. ${ }^{21}$ Other reasons are that TN-LC SLMs offer a coupled amplitude and phase modulation and that they cannot be used as they are for the display of computed phase elements or computed amplitude elements because phase or amplitude errors or both are introduced.

Figure 5 shows the simulation of the reconstruction produced by such displays. We computed an eightlevel pure-amplitude DOE and simulated dephasing produced by displaying the DOE on a $2 \pi$ spiral domain like the one shown in Fig. 6(a).

Such poor performance clearly shows that a full study on spiral domains is necessary. 

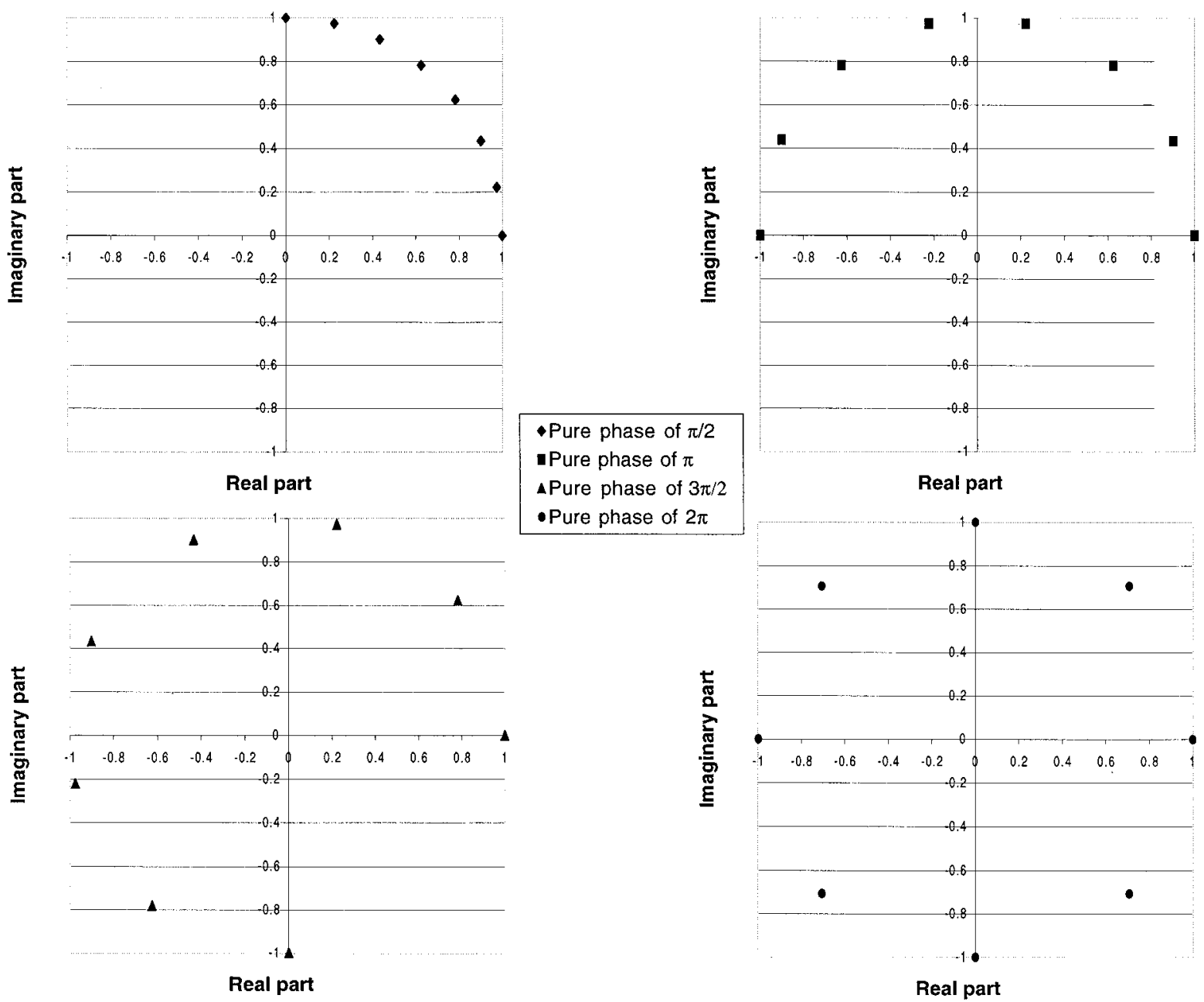

(a)

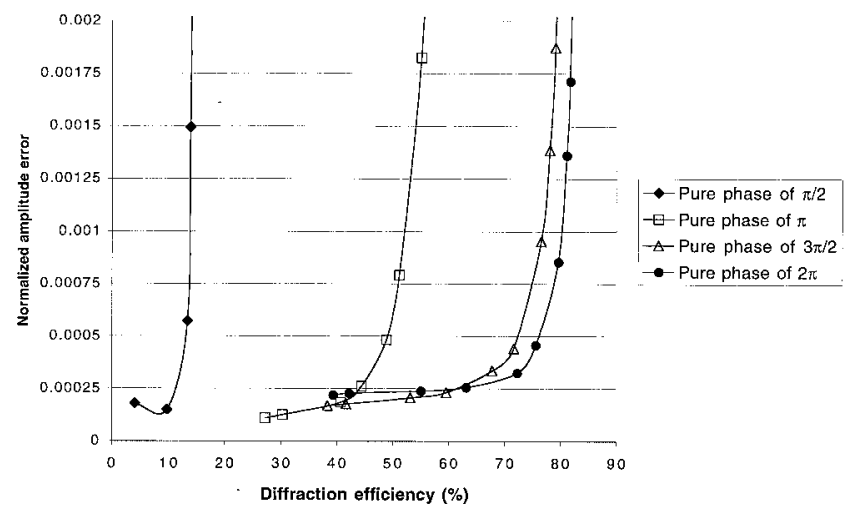

(b)

Fig. 4. (a) Four eight-level phase domains for different maximum phase modulation and (b) the corresponding OCCs.

\section{Coupled Phase and Amplitude Domains}

Here we use four spiral domains [Fig. 6(a)] whose respective maximum modulations are the same as for the former pure-phase domains. As for phase domains, the OCCs [Fig. 6(b)] show that efficiency increases with the phase modulation. However, the efficiency values are not as high as the ones obtained with the equivalent pure-phase domains. This is ex- plained by the fact that the central order always appears with spiral domains because of their amplitude modulation, whatever the maximum phase modulation. But what is important in terms of reconstruction efficiency is the decreasing energy of this central order when the phase modulation increases. The reason for it is that the distribution of the quantization points is more uniform in the complex plane. 


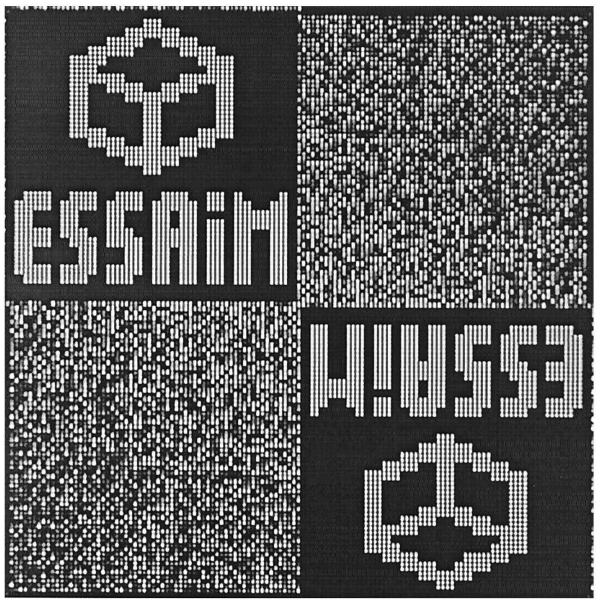

(a)

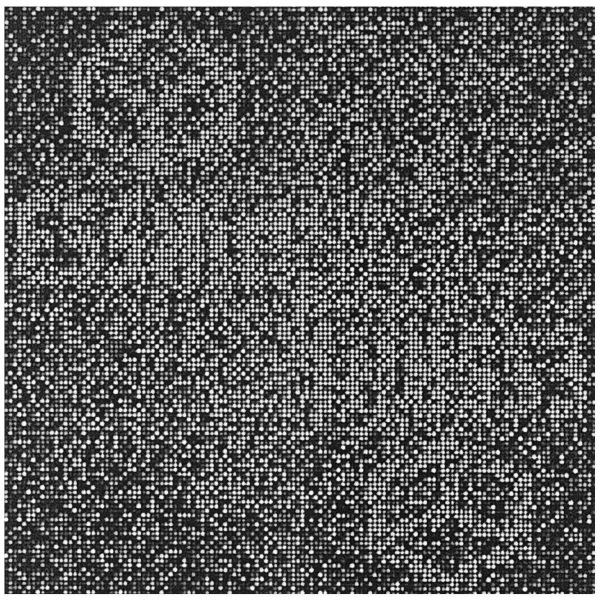

(b)

Fig. 5. (a) Simulation of the reconstruction of an eight-level pureamplitude DOE, difficult to implement in practice. (b) Simulation of the reconstruction of the same DOE when displayed on a SLM with a $2 \pi$ spiral-coding domain.

Finally, we note that the good trade-off is obtained with the $2 \pi$ spiral domain, with an efficiency range from $20 \%$ to $35 \%$ and a $\mathrm{SNR}_{\mathrm{a}}$ range from 10,000 to 1,400 .

Here we note that spiral domains show a trade-off between pure-amplitude domains and pure-phase domains with a high $\mathrm{SNR}_{\mathrm{a}}$ and medium efficiency. In this case, maximum dephasing plays an important role: An increase in the maximum dephasing from $3 \pi / 2$ to $2 \pi$ allows diffraction efficiency to be nearly doubled.

\section{B. Comparison between Domains}

A first comparison between the various spiral-coding domains and the pure-amplitude domains (Fig. 7) clearly shows that the spiral domain limited to $\pi / 2$ leads to efficiency values and $\mathrm{SNR}_{\mathrm{a}} \mathrm{s}$ that locate it between the eight levels of amplitude domain and the binary-amplitude domain. The binary domain has extremely poor coding capabilities because it has only

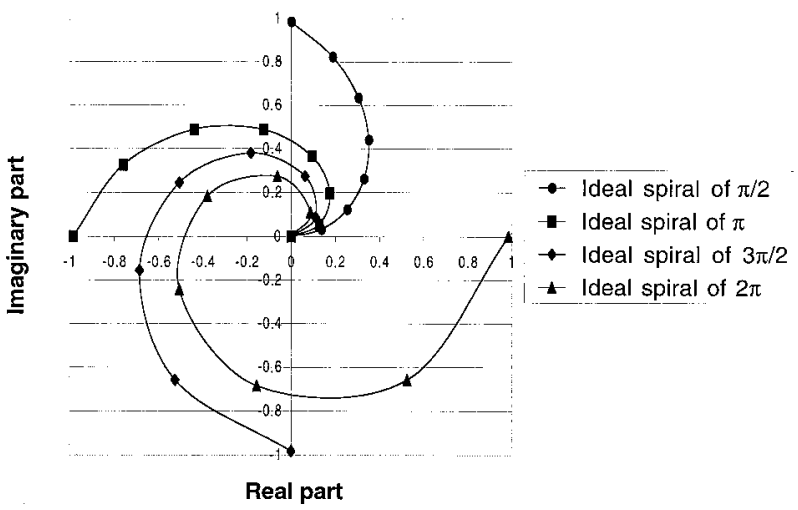

(a)

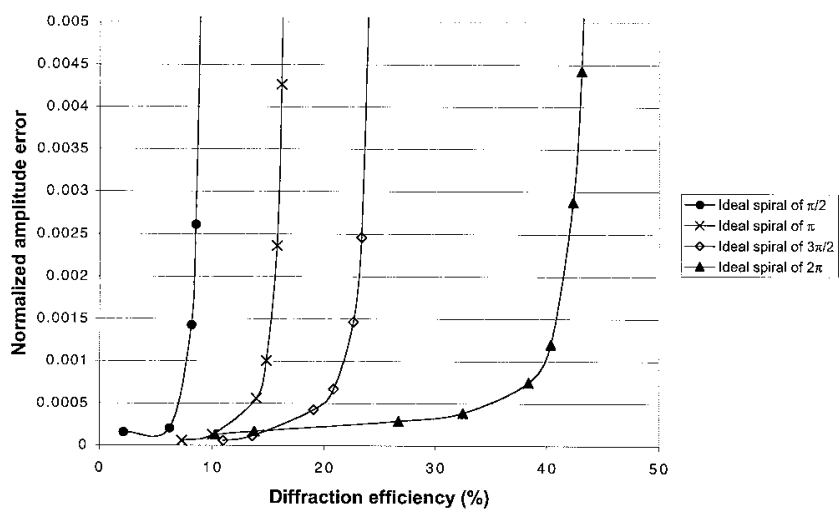

(b)

Fig. 6. (a) Ideal spiral domains and (b) their corresponding OCCs.

two levels; therefore its behavior differs significantly from the other domains.

Thus this spiral-coding domain limited to $\pi / 2$ does not offer a significant gain in diffraction efficiency compared with binary-amplitude quantization but rather offers a gain in terms of $\mathrm{SNR}_{\mathrm{a}}$, allowing a more uniform reconstruction. The increase in the maximum phase modulation of the coding domain allows an increase in efficiency obtained while preserving a high $\mathrm{SNR}_{\mathrm{a}}$. We can thus conclude that a spiral do-

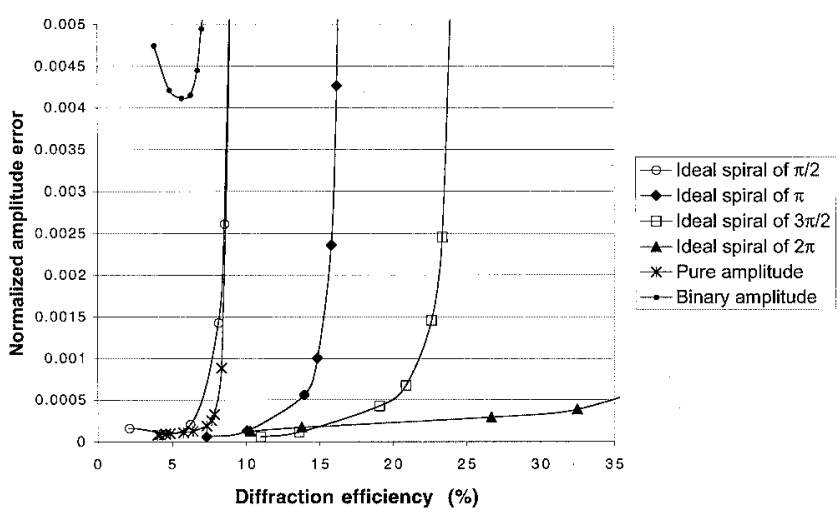

Fig. 7. Comparison between the OCCs of the amplitude domains (binary and eight levels) and of the spiral domains. 


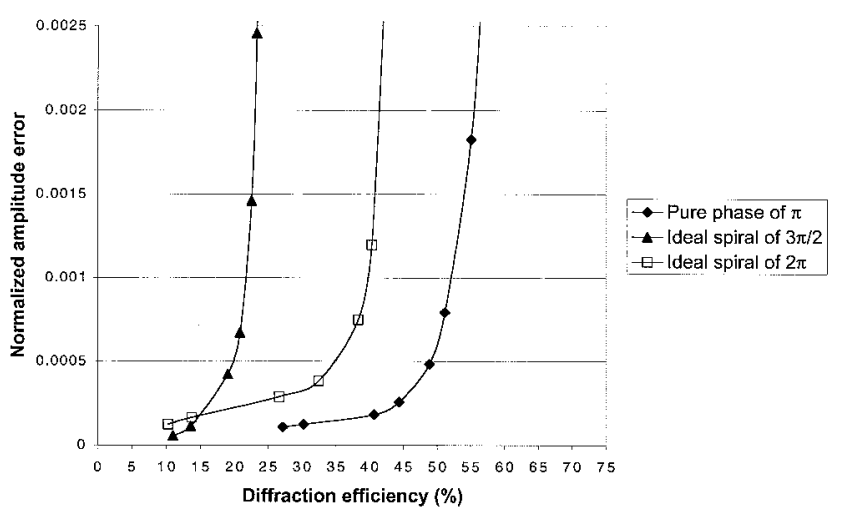

Fig. 8. Comparison between the OCCs of some spiral domains with the one of pure $\pi$ phase domains.

main, with a modulation equal to or larger than $\pi$, is more interesting than a pure-amplitude domain.

But spiral domains and pure-phase domains must also be compared. The fact is that the $2 \pi$ purephase domain provides the best results, with diffraction efficiency reaching $80 \%$ (Fig. 4). In contrast, the $2 \pi$ spiral domain shows maximum efficiency of only $50 \%$ (Fig. 6) and provides a trade-off between pure amplitude and pure phase: The OCC relative to the $2 \pi$ spiral domain lies between the OCC produced by a pure-amplitude modulation and a purephase modulation. However, the SVGA1 TN-LC SLM provides a maximum phase modulation of only $3 \pi / 2$. Therefore it is important to find the domains of this SLM that are best suited for the implementation of dynamic DOEs.

In Fig. 8 the $3 \pi / 2$ spiral clearly shows less diffraction efficiency $(9 \%-20 \%)$ than the $2 \pi$ spiral domain $(9 \%-40 \%)$. Furthermore, this latter domain produces lower quality DOEs than the $\pi$ phase modulation domain that leads to a variation in efficiency from $27 \%$ to $55 \%$. This fact is also shown in Fig. 8 in which the gap of diffraction efficiency is $25 \%$ between the OCCs of these two domains.

When displaying a DOE on a TN-LC SLM, the search for high dephasing may not be a good solution, whereas the need for quite a constant amplitude clearly appears to be the most important factor to get an interesting compromise between the $\mathrm{SNR}_{\mathrm{a}}$ and $\eta$.

\section{Experimental Domains}

The comparisons made in Subsections 4.A and 4.B remain theoretical because such domains are not always experimentally available. Using our Jones model from Section 3, we selected three experimental domains that are close to their equivalent theoretical ones [Fig. 9(a)].

The $\left(20^{\circ}, 80^{\circ}\right)$ domain corresponds to a phasemostly mode with maximum dephasing of $3 \pi / 2$. With our SLM, an amplitude-mostly mode is difficult to reach. For a $\left(0^{\circ},-90^{\circ}\right)$ configuration, a strong coupling between phase and amplitude is obtained.

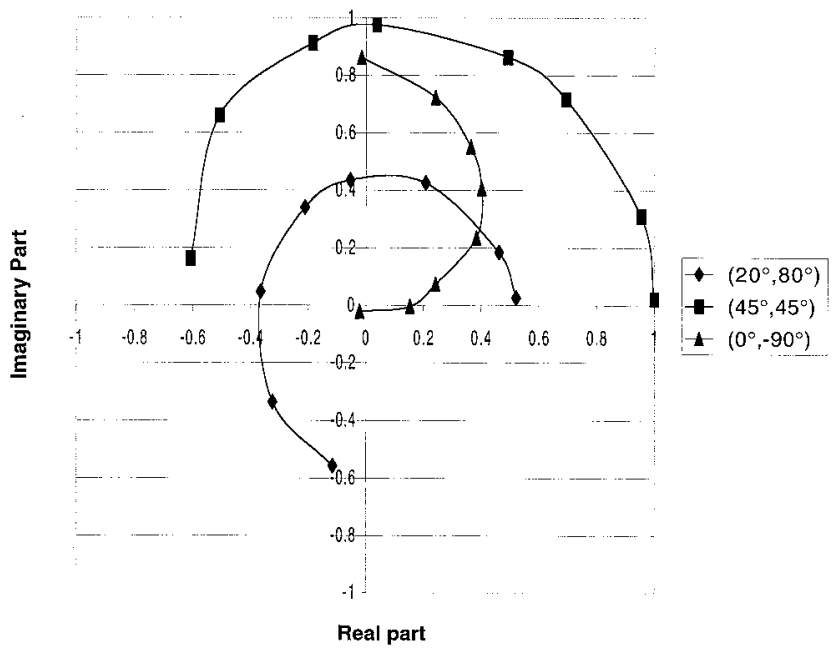

(a)

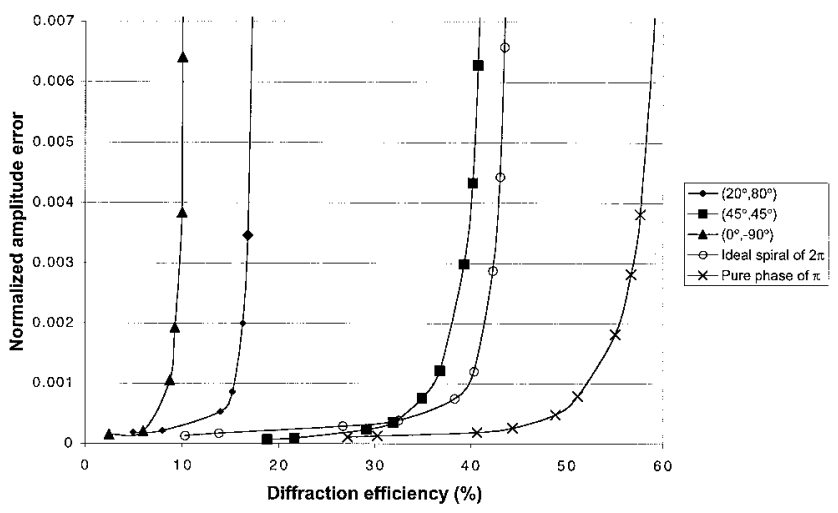

(b)

Fig. 9. (a) Selected experimental domains at $633 \mathrm{~nm}$ and (b) their corresponding OCCs compared with theoretical ones.

The $\left(45^{\circ}, 45^{\circ}\right)$ configuration corresponds to a phasemostly mode with maximum dephasing of $\pi$.

In all the cases [Fig. 9(b)] the signal-to-noise ratio is high and gives good values from 10,000 for low efficiency values to 100 for higher efficiency values. As was said previously, the end (toward $\mu=1$ ) of these OCCs is not usable because in these zones the error in the reconstruction is high. In some cases the whole coding domain is not worth exploiting if it offers reduced low maximum diffraction efficiency (a few percent). It is the case of the $\left(0^{\circ},-90^{\circ}\right)$ domain that hardly reaches $10 \%$ efficiency. These results are in good agreement with the simulations of Subsection 4.A.

However, with our SVGA TN-LC SLM, high dephasing is obtained at the expense of reduced transmittance, and the constraints imposed by the final application will decide if a phase modulation is preferable to a coupled amplitude and phase modulation. ${ }^{22}$

The phase-mostly $\left(20^{\circ}, 80^{\circ}\right)$ configuration then shows a mean transmittance of 0.25 in intensity, resulting in a rather poor diffraction efficiency, hardly reaching 15\%, as shown in Fig. 9(b). For instance, 


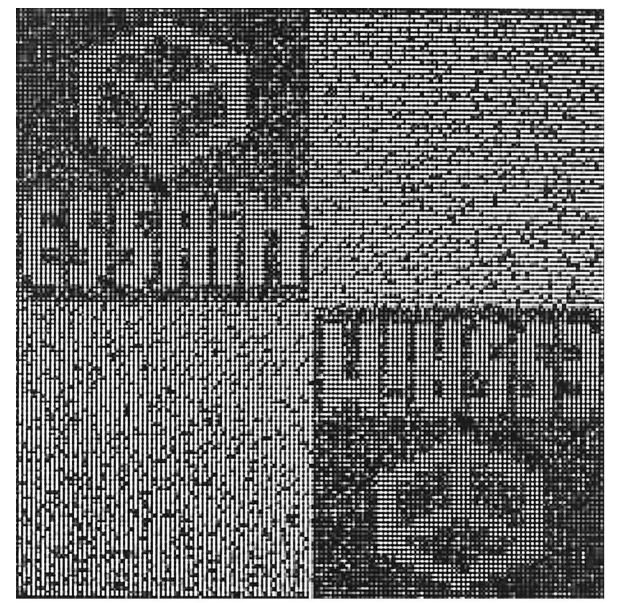

(a)

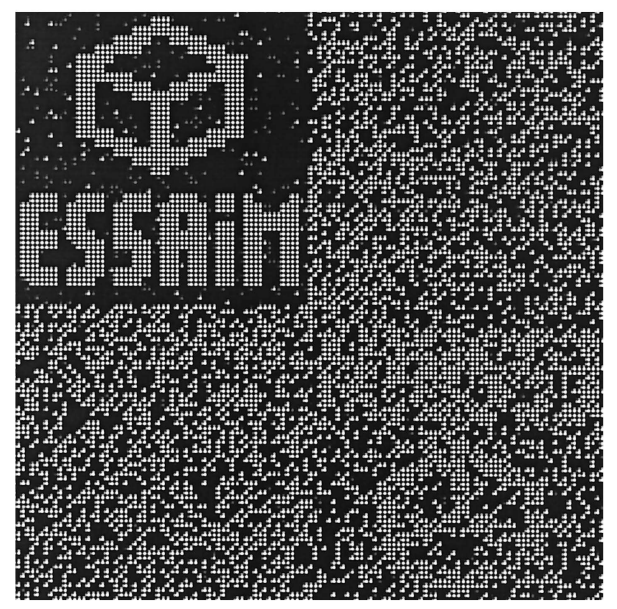

(c)

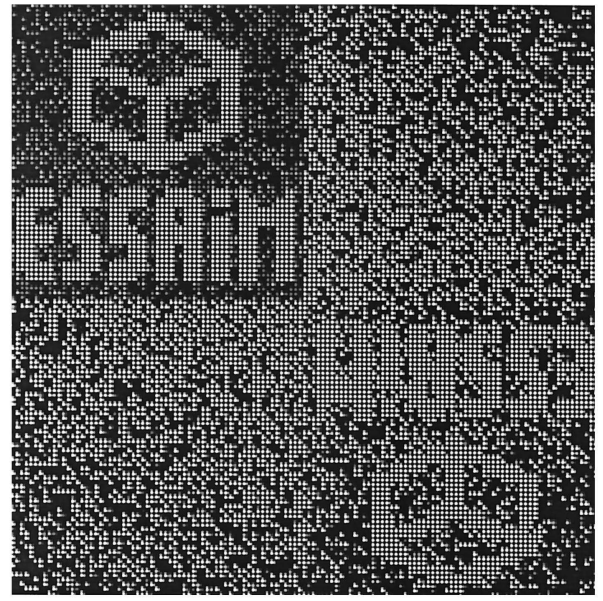

(b)

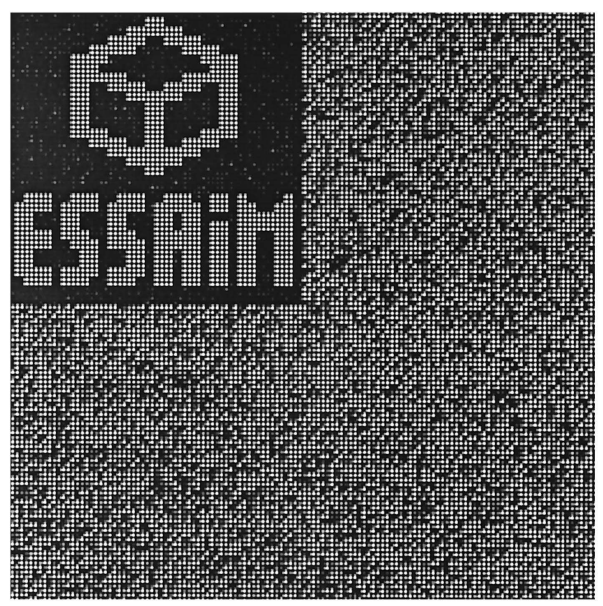

(d)

Fig. 10. Simulations of DOEs designed with (a) binary-amplitude domain, (b) the $\left(0^{\circ},-90^{\circ}\right)$ domain, (c) the $\left(45^{\circ}, 45^{\circ}\right)$ domain, and $(\mathrm{d})$ the $\left(20^{\circ}, 80^{\circ}\right)$ configurations.

the phase-mostly mode reduced to a semicircle $\left[\left(45^{\circ}\right.\right.$, $\left.45^{\circ}\right)$ ] will be preferred because its transmittance is approximately 0.8 and its diffraction efficiency is close to the efficiency given by the theoretical purephase modulation domain (a maximum of 35\%), thus confirming the possibility of using our SLM in this $\pi$ modulation mode.

This study allows us to choose the best coding domains. DOEs coded on such domains can then be computed. We will provide optical reconstructions in Subsection 4.D.

\section{Optical Reconstructions}

The reconstructions provided in this subsection were obtained by application of the former coding domains. We first computed $128 \times 128$ pixels DOEs, which were replicated $6 \times 4$ times before being displayed on the SLM. The size of the original desired reconstruction is $64 \times 64$ pixels instead of $32 \times 32$ in the previous subsections because it is easier to observe the performance of the DOE when the reconstruction is larger.
The simulations (Fig. 10) and the optical reconstructions (Fig. 11) are in good agreement. We clearly see the evolution of the conjugate image when the phase modulation of the coding domain increases from 0 to approximately $3 \pi / 2$.

We verified the diffraction efficiency values of the different DOEs designed with these four coding domains and reported the results in Table 3. They first confirm (result \#1) that the binary domain provides acceptable performance. They also prove that computation for theoretical pure-amplitude multilevel domain (result \#2, described in Subsection 4.A.2) provides poor performance when the DOE is displayed on a SLM (results \#3 and \#4). Results \#6 to \#8 confirm that the design that takes account of the coding domain constraints provides the best performance. Among these latter coding domains, we can select the $\left(45^{\circ}, 45^{\circ}\right)$ coding domain (result \#7) that provides a high efficiency and is close to the one provided by the spiral theoretical domain (result \#5). The reconstruction accuracy provided by the $\left(45^{\circ}\right.$, $45^{\circ}$ ) coding domain, as shown in Fig. 11(c), is satis- 


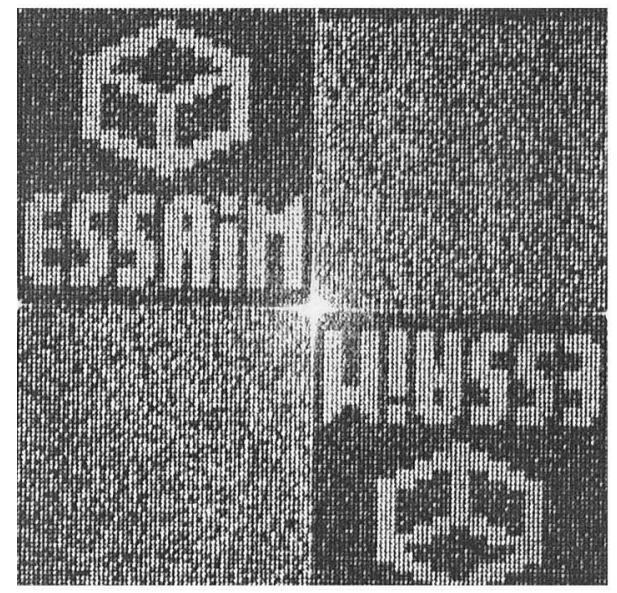

(a)

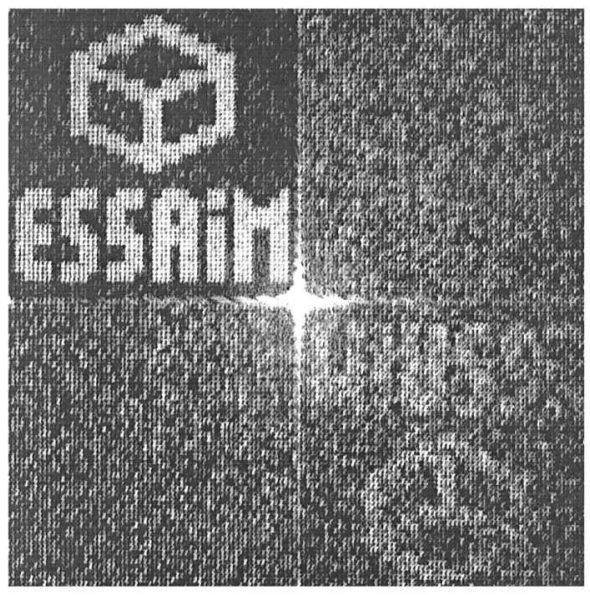

(c)

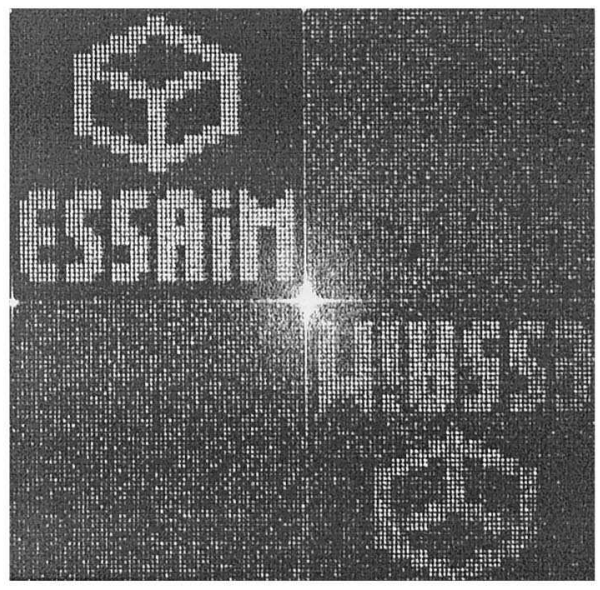

(b)

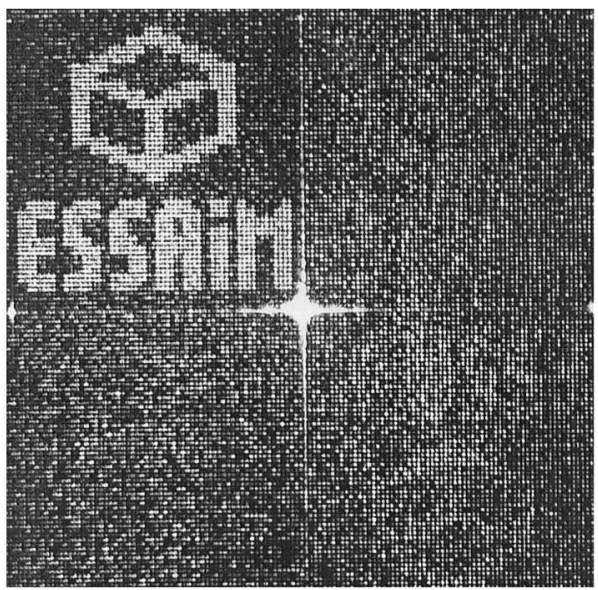

(d)

Fig. 11. Example of optical reconstructions performed with (a) binary-amplitude domain, (b) the $\left(0^{\circ},-90^{\circ}\right)$ domain, $(\mathrm{c})$ the $\left(45^{\circ}, 45^{\circ}\right)$ domain, and (d) the $\left(20^{\circ}, 80^{\circ}\right)$ configurations.

factory. These results emphasize the fact that exploring a coding domain with a shape closed to the $\pi$ pure phase leads to the best possible diffraction efficiency values and therefore proves to be the best compromise when a $2 \pi$ phase domain cannot be reached. However, if we want to remove the conjugate image almost completely, the use of a $3 \pi / 2$ modulation domain is required.

Table 3. Measurement of Relative Diffraction Efficiency of DOEs Computed with Several Coding Domains

\begin{tabular}{|c|c|c|c|c|c|}
\hline \multirow[b]{2}{*}{ Result \# } & \multirow[b]{2}{*}{ Coding Domain } & \multicolumn{3}{|c|}{ Simulations } & \multirow{2}{*}{$\begin{array}{c}\text { Experiments } \\
\begin{array}{c}\text { First-Order Energy } \\
\left(\mu \mathrm{W} / \mathrm{cm}^{2}\right)\end{array}\end{array}$} \\
\hline & & $\begin{array}{c}\text { Diffraction } \\
\text { Efficiency }(\%)\end{array}$ & $\mathrm{SNR}_{\mathrm{a}}$ & CPU Time & \\
\hline 1 & Binary amplitude [Figs. 10(a), 11(a)] & 6.61 & 16 & $15 \mathrm{~min}$ & 16.7 \\
\hline 2 & Eight amplitude levels [Fig. 5(a)] & 3.87 & 253 & $17 \mathrm{~min}$ & \\
\hline 3 & $\begin{array}{l}\text { Eight amplitude levels after dephasing of } \\
2 \pi \text { [Fig. } 5(\mathrm{~b})]\end{array}$ & 28.61 & 1 & $17 \mathrm{~min}$ & \\
\hline 4 & $\begin{array}{l}\text { Eight amplitude levels with SLM in }\left(0^{\circ} \text {, }\right. \\
\left.90^{\circ}\right) \text { configuration }\end{array}$ & 4.71 & 12 & $17 \mathrm{~min}$ & 9.5 \\
\hline 5 & Ideal spiral of $2 \pi$ & 32.5 & 2,600 & $1.5 \mathrm{~h}$ & \\
\hline 6 & $\begin{array}{l}\text { Eight levels of the }\left(0^{\circ},-90^{\circ}\right) \text { configuration } \\
\quad[\text { Figs. } 10(\mathrm{~b}), 11(\mathrm{~b})]\end{array}$ & 8.2 & 93 & $2 \mathrm{~h}$ & 11.9 \\
\hline 7 & $\begin{array}{l}\text { Eight levels of the }\left(45^{\circ}, 45^{\circ}\right) \text { configuration } \\
\text { [Figs. 10(c), 11(c)] }\end{array}$ & 31.94 & 2,824 & $2 \mathrm{~h}$ & 27 \\
\hline 8 & $\begin{array}{l}\text { Eight levels of the }\left(20^{\circ}, 80^{\circ}\right) \text { configuration } \\
\quad \text { Fig. } 10(\mathrm{~d}), 11(\mathrm{~d})]\end{array}$ & 12 & 132 & $2 \mathrm{~h}$ & 13.3 \\
\hline
\end{tabular}




\section{Conclusion}

We have studied the properties of the coupling between amplitude and phase in the implementation of DOEs. The investigations performed through simulations, with spiral domains, show that such a phenomenon allows a user to adjust the compromise between two well-known behaviors that cannot be obtained in a dynamic implementation-one provided by amplitude coding and the other one by phase-only coding. We have also investigated the influence of maximum dephasing of the domain. This parameter plays a significant role for phase domains, as well as for amplitude-phase domains. Our conclusion is that if we use the TN-LC SLM, we can obtain a coding domain close to a phase domain modulating at $\pi$ or more and providing high transmittance; the performance of the computed DOE with such a domain will be close to the theoretical performance and will offer a good trade-off: high efficiency (within the limits of the coding domain) and acceptable reconstruction accuracy. This is proven by the production of optical reconstructions on a SVGA TN-LC SLM. Actually, we have shown that using high-quality DOEs displayed onto TN-LC SLMs is relevant when their characteristics are completely taken into account.

\section{References}

1. V. Laude and C. Dirson, "Liquid-crystal active lens: application to image resolution enhancement," Opt. Commun. 163, 72-78 (1999).

2. CRL Opto, "Miniatures LCDs," http://www.crlopto.com/wop/ lc-prods.htm (1999).

3. V. Laude and P. Réfrégier, "Multicriteria characterization of coding domains with optimal Fourier SLM filters," Appl. Opt. 33, 4465-4471 (1994).

4. D. A. Gregory, "Real-time pattern recognition using a modified liquid crystal television in a coherent optical correlator," Appl. Opt. 25, 467-469 (1986).

5. L. G. Neto, D. Roberge, and Y. Sheng, "Full-range, continuous, complex modulation by the use of two coupled-mode liquidcrystal televisions," Appl. Opt. 35, 4567-4576 (1996).

6. L. G. Neto, D. Roberge, and Y. Sheng, "Programmable optical phase-mostly holograms with coupled-mode modulation liquid crystal television," Appl. Opt. 34, 1944-1950 (1995).

7. P. Réfrégier, "Filter design for optical pattern recognition: multicriteria optimization approach," Opt. Lett. 15, 854-856 (1990).

8. L. Legeard, P. Réfrégier, and P. Ambs, "Multicriteria optimal- ity for iterative encoding of computer-generated holograms," Appl. Opt. 36, 7444-7449 (1997).

9. M. A. Seldowitz, J. P. Allebach, and D. W. Sweeney, "Synthesis of digital holograms by direct binary search," Appl. Opt. 26, 2788-2798 (1987).

10. L. Bigué and P. Ambs, "Optimal multicriteria approach to the iterative Fourier transform algorithm," Appl. Opt. 40, 58865893 (2001).

11. Z. Zhang, G. Lu, and F. T. S. Yu, "Simple method for measuring phase modulation in liquid crystal televisions," Opt. Eng. 33, 3018-3022 (1994).

12. M. Yamauchi and T. Eiju, "Optimization of twisted nematic liquid crystal panels for spatial light phase modulation," Opt. Commun. 115, 19-25 (1995).

13. J. Colin, "Corrélation optique photoréfractive haute cadence à transformée de Fourier conjointe," Thesis, Université Paris 6, Paris, France (1998).

14. C. Soutar and K. Lu, "Determination of the physical properties of an arbitrary twisted-nematic liquid crystal cell," Opt. Eng. 33, 2704-2712 (1994).

15. I. Labastida, A. Carnicer, E. Martin-Badosa, S. Vallmitjana, and I. Juvells, "Optical correlation by use of partial phase-only modulation with VGA liquid-crystal displays," Appl. Opt. 39, $766-769$ (2000).

16. K. Lu and B. E. A. Saleh, "Theory and design of the liquid crystal TV as an optical phase modulator," Opt. Eng. 29, 240 246 (1990).

17. C. R. Fernández-Pousa, I. Moreno, N. Bennis, and C. GómezReino, "Generalized formulation and symmetry properties of reciprocal nonabsorbing polarization devices: application to liquid-crystal displays," J. Opt. Soc. Am. A 17, 2074-2080 (2000).

18. S. E. Monroe, M. J. Rollins, and R. D. Juday, "Advances in full-face full-complex SLM characterization," in Optical Pattern Recognition XII, D. P. Casasent and T.-H. Chao, eds., Proc. SPIE 4387, 68-77 (2001).

19. S. E. Broomfield, M. A. A. Neil, and E. G. S. Paige, "Programmable multiple-level phase modulation that uses ferroelectric liquid-crystal spatial light modulators," Appl. Opt. 34, 66526665 (1995).

20. J. A. Davis, P. Tsai, D. M. Cottrell, T. Sonehara, and J. Amako, "Transmission variations in liquid crystal spatial light modulators caused by interference and diffraction effects," Opt. Eng. 38, 1051-1057 (1999).

21. N. Mukohzaka, N. Yoshida, H. Toyoda, Y. Kobayashi, and T. Hara, "Diffraction efficiency analysis of a parallel-aligned nematic-liquid-crystal spatial light modulator," Appl. Opt. 33, 2804-2811 (1994).

22. C. Stolz, L. Bigué, and P. Ambs, "High-resolution multilevel computer generated holograms on a TN LCD spatial light modulator," in Diffractive Optics, F. Wyrowski and J. Turunen, eds., Vol. 22 of EOS Topical Meetings Digest Series (European Optical Society, Hanover, Germany), pp. 215-216 (1999). 